\title{
Clinical and Functional Characterization of a Human ORNT1 Mutation (T32R) in the Hyperornithinemia-Hyperammonemia- Homocitrullinuria (HHH) Syndrome
}

\author{
JOSÉ A. CAMACHO, REBECCA MARDACH, NATALIA RIOSECO-CAMACHO, EDUARDO RUIZ-PESINI, OLGA DERBENEVA, \\ DARIO ANDRADE, FRANK ZALDIVAR, YONG QU, AND STEPHEN D. CEDERBAUM
}

\begin{abstract}
Department of Pediatrics [J.A.C., N.R.-C., E.R.-P., O.D., F.Z.], Division of Human Genetics, University of California-Irvine and Center for Molecular and Mitochondrial Medicine and Genetics, Irvine, California, 92697; Division of Medical Genetics [R.M., Y.Q.], Kaiser Permanente Medical Center, Los Angeles, California, 90027;Departments of Psychiatry, Pediatrics and Human Genetics [S.D.C.], David Geffen School of Medicine at University of California-Los Angeles, Los Angeles, California, 90095; Department of Pediatrics [J.A.C., N.R.-C., D.A.], University of Oklahoma Health Sciences Center, Oklahoma City, Oklahoma, 73130
\end{abstract}

\begin{abstract}
We studied two related families (HHH013 and HHH015) with the hyperornithinemia-hyperammonemia-homocitrullinuria $(\mathrm{HHH})$ syndrome, a disorder of the urea cycle and ornithine degradation pathway, who have the same novel ornithine transporter (ORNT1) genotype (T32R) but a variable phenotype. Both HHH015 patients are doing well in school and are clinically stable; conversely, the three affected HHH013 siblings had academic difficulties and one suffered recurrent episodes of hyperammonemia and ultimately died. Overexpression studies revealed that the product of the ORNT1$\mathrm{T} 32 \mathrm{R}$ allele has residual function. Ornithine transport studies in HHH015 fibroblasts, however, showed basal activity similar to fibroblasts carrying nonfunctional ORNT1 alleles. We also examined two potential modifying factors, the ORNT2 gene and the mitochondrial DNA lineage (haplogroup). Haplogroups, associated with specific diseases, are hypothesized to influence mitochondrial function. Results demonstrated that both HHH015 patients are heterozygous for an ORNT2 gain of function polymorphism and belong to haplogroup A whereas the HHH013 siblings carry the wild-type ORNT2 and are haplogroup $\mathrm{H}$. These observations suggest that the ORNT1 genotype cannot predict the phenotype of HHH patients. The reason for the phenotypic variability is unknown, but factors such as redundant transporters and mitochondrial lineage may contribute to the neuropathophysiology of HHH patients. (Pediatr Res 60: 423-429, 2006)
\end{abstract}

$\mathrm{T}$ The HHH syndrome (OMIM \#238970) is an autosomal recessive disorder of the urea cycle and ornithine degradation pathway caused by the deficient transport of ornithine across the inner mitochondrial membrane $(1,2)$. The gene defective in $\mathrm{HHH}$ syndrome is the mitochondrial ornithine transporter (ORNT1) that is localized in the q14.1 region of Ch13 and is a member of the MCF of proteins that includes the uncoupling protein, carnitine/acyl-carnitine translocase, and the ADP/ATP transporter $(3,4)$. The human ORNT1 gene

Received December 1, 2005; accepted June 8, 2006.

Correspondence: José Angel Camacho, M.D., Center for Molecular and Mitochondrial Medicine \& Genetics (MAMMAG), 2036 Hewitt Hall, University of California, Irvine, Irvine, CA 92697-3940; e-mail: jcamacho@uci.edu

This work was supported by a Robert Wood Johnson Foundation Harold Amos Faculty Development Award and an Oklahoma Center for the Advancement of Science and Technology grant (HR01-018) to J.A.C., M01RR00827-29 grant to F.Z., and a PHS grant (HD-06576) to S.D.C.

DOI: 10.1203/01.pdr.0000238301.25938.f5 is expressed in the periportal hepatocytes, which contain the urea cycle pathway, and in the pericentral hepatocytes and skin fibroblasts that express the ornithine degradation pathway $(1,2)$. Physiologically, ORNT1 allows ornithine to serve as a substrate for the ornithine transcarbamylase (OTC) and ornithine amino transferase (OAT) reactions that produce citrulline and the two amino acids, glutamate and proline. In vitro studies have demonstrated that ORNT1 transports ornithine, lysine, and arginine across the inner mitochondrial membrane in exchange for a hydrogen ion and citrulline (5).

Biochemically, HHH syndrome is characterized by persistent elevation of plasma ornithine, episodic or postprandial hyperammonemia, and the urinary excretion of homocitrulline and orotic acid (1). The homocitrulline is believed to be the product of transcarbamoylation of lysine whereas the orotic aciduria occurs secondary to decreased OTC activity. Numerous studies have clearly demonstrated a link between hyperammonemia and CNS pathology in urea cycle disorders. However, little is known about the mechanisms by which chronic accumulation of ornithine in the intracellular compartment or decrease of its transport across the mitochondrial membrane impairs the brain function of HHH patients $(1,4,5)$.

Although there are reports of $\mathrm{HHH}$ patients presenting with neonatal hyperammonemia similar to patients with other urea cycle disorders, the onset of symptoms in $\mathrm{HHH}$ patients is usually during infancy to early school years (1). We believe this difference in phenotype may in part be related to the existence of genes with redundant function to ORNT1 such as the second ornithine transporter, ORNT2, previously shown to be a functional retroposon (4-6). Presenting complaints may include unexplained seizure activity, vomiting, developmental

Abbreviations: HHH, hyperornithinemia-hyperammonemia-homocitrullinuria; MCF, mitochondrial carrier family of proteins; mtDNA, mitochondrial DNA; ORNT1, human mitochondrial ornithine transporter 1; ORNT2, human mitochondrial ornithine transporter 2; ROS, reactive oxygen species 
delay, mental retardation, academic difficulties, and/or gait disturbances. The cognitive development of HHH patients can vary from low normal intelligence to severe mental retardation. Liver involvement is commonly seen as elevated liver enzymes and prolonged coagulation studies (1). In general, $\mathrm{HHH}$ patients are maintained on a protein restricted diet, supplemental citrulline, and sodium phenylbutyrate (1).

Although several reports have described different ORNT1 mutant alleles, functional studies have not demonstrated a correlation between protein function and clinical course (4,5,7-9). We believe that factors influencing the phenotypic expression of ORNT1 deficiency may include gene redundancy as well as maternally inherited differences in mitochondrial physiology related to mtDNA lineage (haplogroup) $(10,11)$. In this report, we describe two related HHH families who have the same ORNT1 genotype (T32R) but a variable phenotype. Given the phenotypic variability of our group of patients, we also examined two factors that may potentially affect their clinical course, the ORNT2 genotype and mitochondrial haplogroup.

\section{SUBJECTS AND METHODS}

\section{Subjects}

We studied two related families of Mexican ancestry where the maternal grandfather of the HHH015 proband (Case 1) and the paternal grandfather of the HHH013 proband (Case 3) are first cousins (Fig. 1). Studies were performed after informed consent was obtained in accordance with approved Kaiser Permanente human institutional review board protocols. The parents of the affected probands originally migrated from the same small city (population 26,000 ) in North-Central Mexico. The diagnosis of HHH syndrome was based on the significant elevation of plasma ornithine, glutamine, and ammonia in addition to increased urinary excretion of orotic acid (Table 1) (1). For this study, the five affected patients and their parents consented to donating blood for DNA and lymphocyte cultures, whereas only the two HHH015 siblings and their father consented to a skin biopsy. Clinical data are summarized in Table 1. Kaiser Permanente psychologists administered psychometric evaluations.

\section{Methods}

PCR amplification of human ORNT1 and ORNT2 from genomic DNA. We isolated genomic DNA from peripheral blood as previously published $(4,6)$.

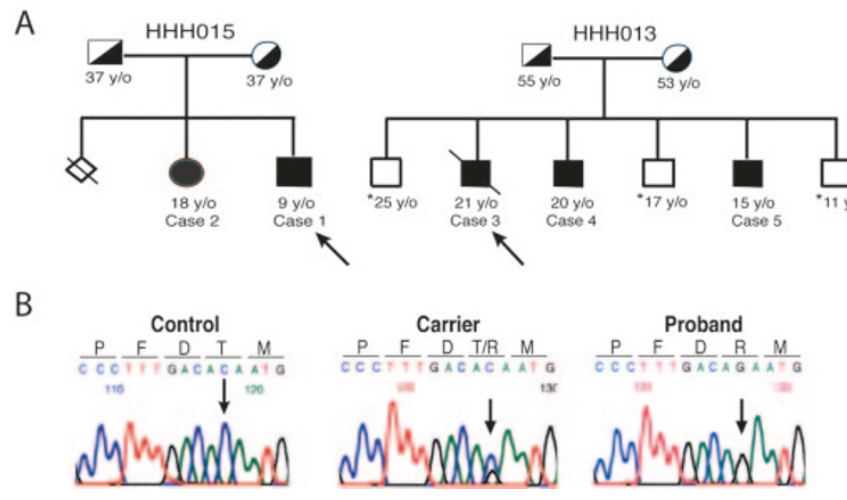

Figure 1. Pedigree and sequence of two related HHH families homozygous for the ORNT1-T32R allele. (A) Separate pedigrees of both HHH013 and HHH015 families. The maternal grandfather of the HHH015 siblings and the paternal grandfather of the HHH013 brothers are first cousins. Arrows indicate proband in each family. All members of these two families were genotyped except the three nonaffected siblings (*) from the HHH013 family that have normal plasma ornithine, glutamine, ammonia, and urinary excretion of orotic acid. (B) Sequence of genomic DNA showing the C95G change in the human ORNT1 ORF from a control, parent (carrier), and affected HHH patient.
Primers were designed to amplify the exon-intron regions covering the ORNT1 open reading frame $(\mathrm{ORF})$, exons 2 through 7, based on genomic clone AL161614 and previously published reports $(4,6,7)$. Results of our scan uncovered a homozygous missense mutation, T32R, in exon 3 . We amplified exon 3 with flanking intronic primers HORNT(+)23 and HORNT(-)35a. This product was purified and sequenced using standard protocols utilizing intronic primers HORNT(+)22 and HORNT(-)21a. To confirm that ORNT1-T32R was a mutation not a polymorphism, we screened exon 3 of the ORNT1 gene of 116 control individuals from different ethnic backgrounds (25 Mayan Indian, 28 Spanish, 35 French Canadian, 15 U.S. Hispanic, 8 Caucasian, and 5 African American). The primers and PCR conditions for amplification and sequencing of the human ORNT2 ORF have been previously published (6). Sequences of all new primers used in this study are presented in Table 2 .

Total RNA Isolation and RT-PCR. Total RNA from untransformed cultured control and HHH fibroblasts (HHH015) plus EBV-transformed lymphocytes (HHH013 and HHH015) was isolated as previously reported $(4,6)$. We then isolated poly $\mathrm{A}^{+}$RNA from total RNA utilizing the Oligotex mRNA mini kit (QIAGEN, Valencia, CA). All RNA was treated with RNase-free DNase (Invitrogen, Carlsbad, CA). Total RNA from human liver (Clontech, San Francisco, CA) was used as a positive control in some experiments. We amplified the human ORNT1 message with two sets of overlapping primers: HY(+)1/HYMC $(-) 19$ and HYMC $(+) 27 \mathrm{~b} / \mathrm{HYMC}(-) 26 \mathrm{a}$. The human ORNT2 ORF mRNA was amplified using $5 \mathrm{Q}(+) 11$ and $5 \mathrm{Q}(-)$ 1a primers. The primers used in the OTC and the glyceraldehyde-3-phosphate dehydrogenase (G3PDH) mRNA amplifications are reported elsewhere (6). The One-Step PCR method (QIAGEN) was performed in a single tube with $2 \mu \mathrm{g}$ of total RNA or $0.2 \mu \mathrm{g}$ of polyA + RNA in a total reaction volume of $50 \mu \mathrm{L}$, which included $2 \mu \mathrm{M}$ sense/antisense primers, $1 \times$ reaction buffer with $2.5 \mathrm{mM} \mathrm{Mg}{ }^{+2}, 0.8 \mathrm{mM}$ dNTPs and $2 \mu \mathrm{L}$ of reverse transcriptase/Taq polymerase enzyme mix. We used the following RT-PCR conditions: $50^{\circ} \mathrm{C} 30 \mathrm{~min}(\times 1) ; 95^{\circ} \mathrm{C} 30 \mathrm{~s}(\times 1) ; 94^{\circ} \mathrm{C} 30 \mathrm{~s}, 58^{\circ} \mathrm{C} 30 \mathrm{~s}, 72^{\circ} \mathrm{C} 30 \mathrm{~s}$ $(\times 35) ; 72^{\circ} \mathrm{C} 5$ min $(\times 1)$. We digested ORNT2 RT-PCR products with $R s a \mathrm{I}$ (New England Biolabs, Beverly, MA) to detect the presence of a mutated site. All ORNT1 and ORNT2 RT-PCR products were purified and sequenced as previously mentioned (6).

Human ORNT1 expression constructs. We generated human ORNT1 wild-type $\mathrm{N}$-myc (EQKLISEEDLL)-tagged construct as previously published (4). We used a QuikChange XL Site-Directed Mutagenesis Kit (Stratagene, La Jolla, CA) to perform the necessary amino acid changes in human ORNT1. The following primers were used to introduce changes in the N-myc-ORNT1 wild-type construct: for ORNT1-T32R, JC147(+) and JC148(-) primers were used; for ORNT1-T32S, JC167(+) and JC168(-) primers were used.

Mitochondrial DNA. We analyzed the mtDNA control (noncoding) region of Case 2 (HHH015) and Case 3 (HHH013) to determine the haplogroup (mtDNA lineage) of our two affected families.

As previously reported by other investigators, we used $15978(+)$ and $725(-)$ primers to amplify and the $16028(+)$ and $\mathrm{H} 112(-)$ primers to sequence (10). Standard PCR conditions for mtDNA amplification were followed $(10,11)$. The resulting mtDNA sequences were analyzed by the SEQUENCHER 4.05 software program and compared with the revised Cambridge Reference Sequence as previously reported (12). To determine the presence of an AluI RFLP, we amplified a 975 bp fragment with L6563(+) and H7497(-) primers corresponding to the cytochrome oxidase subunit I and tRNA-Ser genes.

Transfections, ornithine transport assay, and indirect immunofluorescence. We used established transformed primary fibroblasts that carry the ORNT1-F188 allele (from HHH004 proband) that produces an unstable protein, the wild-type ORNT1 allele (C-5756) and the ORNT1-E180 K allele (from another kindred, HHH010) that produces a nonfunctional protein with normal mitochondrial targeting in our experiments (4). We performed electroporation, the ornithine transport assay, and immunofluorescence as previously described $(4,6)$. For expression studies, we assayed 3 to 6 wells per transfected plasmid and expressed results as mean \pm SEM. In the ornithine incorporation assays, fibroblasts were incubated in the presence of 1.0 $\mu \mathrm{Ci} / \mathrm{mL}$ each of ${ }^{14} \mathrm{C}$-ornithine and ${ }^{3} \mathrm{H}$-leucine. We expressed the ability to incorporate ornithine into protein as glutamate and proline relative to total protein synthesis as ${ }^{14} \mathrm{C} /{ }^{\beta} \mathrm{H}$ ratio. We analyzed data using the unpaired $t$ test and presented our results in graphs as percent increase compared with control (untransfected) cells.

\section{RESULTS}

Human ORNT1 mutation and expression analyses. We used genomic DNA from parents and patients to perform our mutation analyses. Screening of the exonic structure that codes for the ORNT1 ORF and the exon-intron junctions revealed the presence of a $\mathrm{C}$ to $\mathrm{G}$ change in exon 3, which 
Table 1. Summary of clinical and biochemical phenotype of five related HHH patients

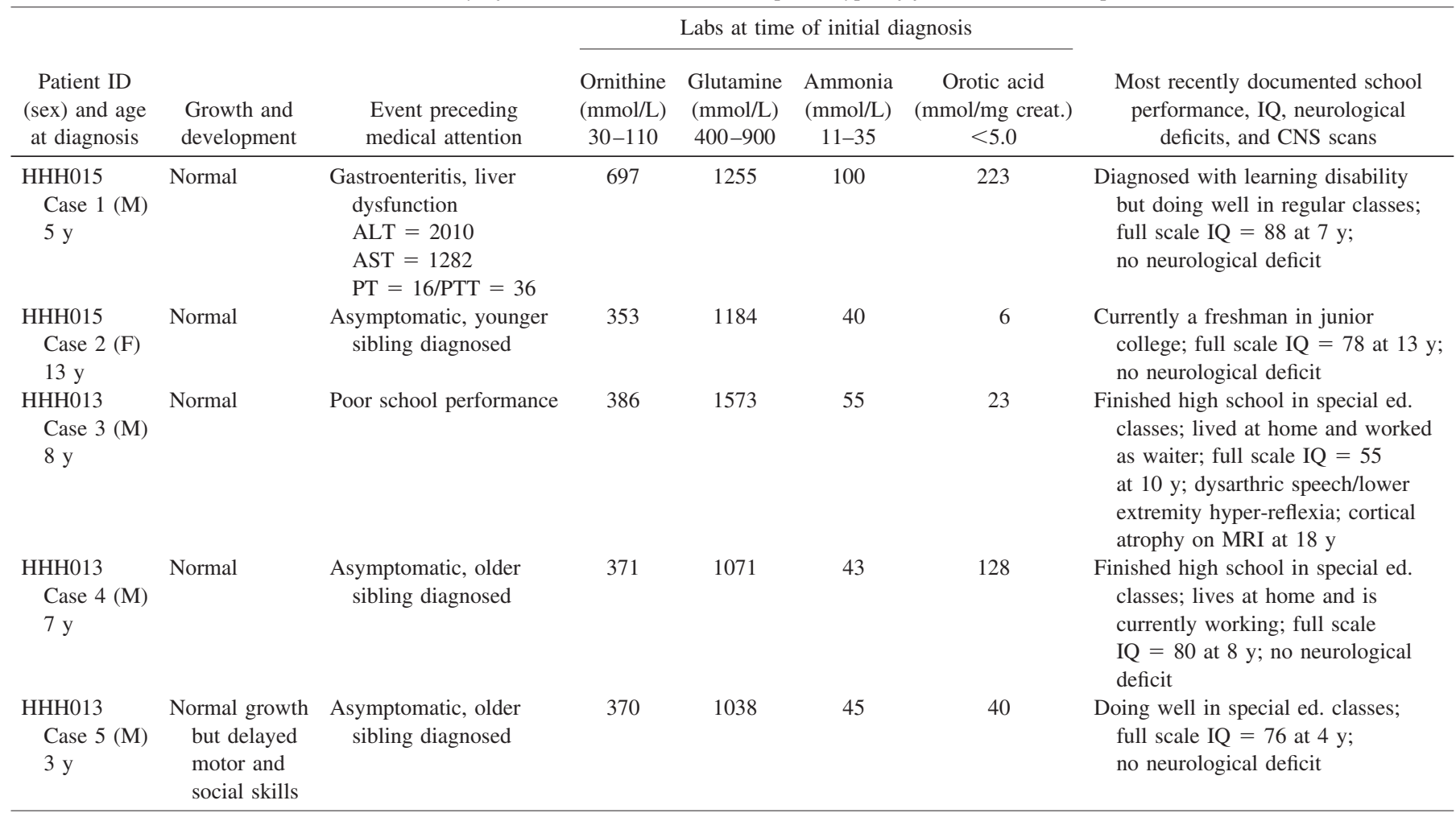

At the time of diagnosis, homocitrulline was not separated from methionine in the urine amino acid chromatogram. However, all five patients had a significant elevation of urine methionine with normal plasma methionine, suggesting urinary excretion of homocitrulline. At the time of diagnosis, all five patients had a normal neurological exam. During the last 3 y, patient HHH013 Case 3 was hospitalized seven times for symptomatic hyperammonemia (needing hemodialysis on occasion) not related to an illness, diet indiscretion, trauma, or infection. Very recently, this patient (HHH013 Case 3) passed away secondary to complications of hyperammonemic encephalopathy. All patients are currently on $1.0 \mathrm{~g} / \mathrm{kg} / \mathrm{d}$ of total protein, citrulline, supplemental Cyclinex-II, and standard doses of sodium phenylbutyrate.

Table 2. Primer combinations for PCR, sequencing, and site directed mutagenesis

\begin{tabular}{|c|c|c|}
\hline Primer ID & Genomic and RT PCR & $\begin{array}{l}\text { Size of PCR } \\
\text { product (bp) }\end{array}$ \\
\hline HORNT $(+) 23$ & GGACATGGCTGGAAGGTTTTG & 581 \\
\hline HORNT( $(-) 35 \mathrm{a}$ & GATGAGGATCTTGCTGTGTTGCC & \\
\hline $\operatorname{HYMC}(-) 19 \mathrm{a}$ & AАTCCCACCAACTCCACCAC & \\
\hline $\operatorname{HYMC}(+) 27 b$ & GCACTTTACTTCGAGAAGTACCAGGC & 471 \\
\hline $\operatorname{HYMC}(-) 26 \mathrm{a}$ & CTTCACACTGGTCTTGTACTCCAAG & \\
\hline $159768(+)$ & CACCATTAGCACGCAAAGCT & 1316 \\
\hline $725(-)$ & GGTGAACTCACTGGAACGGG & \\
\hline L6563(+) & ACCTCAACACCACCTTCTTC & 975 \\
\hline \multirow[t]{2}{*}{$\mathrm{H} 7497(-)$} & TTTGAAAAAGTCATGGAGGCC & \\
\hline & Site directed mutagenesis & \\
\hline $\mathrm{JC} 168(-)$ & Sequencing & \\
\hline HORNT $(+) 22$ & GAGGTTGAAATGAACCGAAGC & \\
\hline $\operatorname{HORNT}(-) 21 \mathrm{a}$ & CATGAGCCACCATGTCTTGCC & \\
\hline $16028(+)$ & TCATGGGGAAGCAGATTTGG & \\
\hline $\mathrm{H} 112(-)$ & ACAGATACTGCGACATAGGG & \\
\hline
\end{tabular}

$(+)$ Denotes forward primer and $(-)$ denotes reverse primer.

All primers are in the $5^{\prime}$ to $3^{\prime}$ sequence. 
caused a threonine to arginine change at codon 32 (T32R). All five patients from both families were homozygous for this T32R change and all parents were found to be carriers (Fig. 1). This single base change does not alter an enzyme restriction site and occurs at nt 95 of the ORNT1 ORF (C95G). As shown in Figure $2 B$, the T32R mutation occurs in the region of the first hydrophilic loop facing the mitochondrial matrix. This region is important since it contains the first of three mitochondrial amino acid sequence motifs characteristic of all MCF proteins (3). Furthermore, $\mathrm{Thr}^{32}$ has been conserved throughout evolution thus suggesting an important role in ORNT1 protein function. Interestingly, this amino acid change, T32R, abolishes a theoretical protein kinase C (PKC) phosphorylation site (Ser/Thr-Xaa-Arg/Lys) (13).

To confirm that the T32R change is a mutation and not a polymorphism, we amplified the human ORNT1 exon 3 from 116 control individuals (35 French Canadian, 28 Spanish, 25 Mayan Indian, 15 U.S. Hispanic, 8 Caucasian and 5 African American). We did not detect the presence of the T32R allele, thus confirming that the T32R change in our group of $\mathrm{HHH}$ patients is a true mutation in the human ORNT1 structural gene.

We next sought to determine whether the $\mathrm{C} 95 \mathrm{G}$ alteration affected the splicing or expression level of the ORNT1 transcript. RT-PCR results shown in Figure 2A (HHH013 Case 3 and his mother) demonstrate no effect of the $\mathrm{C} 95 \mathrm{G}$ change on ORNT1 mRNA content or splicing. Similar findings for

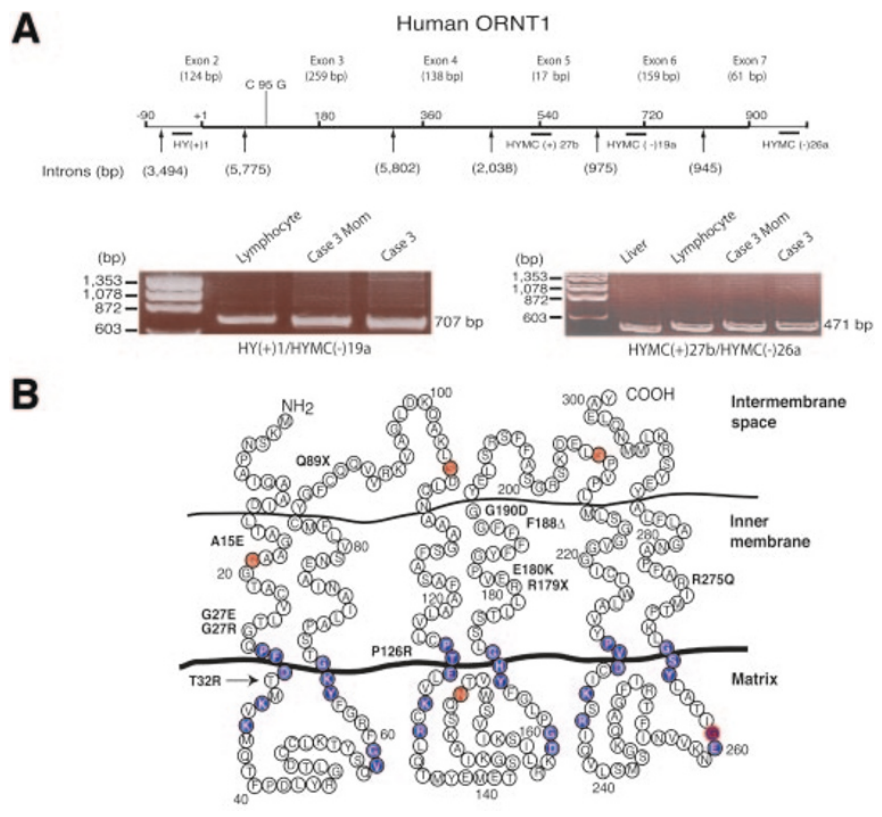

Figure 2. RT-PCR amplification of human ORNT1 and ORNT1 protein structure. (A) Schematic drawing of the ORNT1 gene showing the exonic structure. Arrows designate sites where introns interrupt the $5^{\prime}$ UTR and ORF. Position of the G95C mutation is shown. We used overlapping primer combinations, HY(+)1/HYMC(-)19a and HYMC(+)27b/HYMC(-)26a, to amplify ORNT1 (HHH013 Case 3 and his mother) from human liver (1 $\mu \mathrm{g}$ total RNA) and lymphocytes ( $0.2 \mu \mathrm{g}$ of polyA $\left.{ }^{+} \mathrm{RNA}\right)$. We ran the products on a $3.0 \%$ agarose gel with HaeIII markers. (B) The human ORNT1 protein structure and the position of the T32R change in relation to other published missense and nonsense mutations (5). The mitochondrial carrier signature motif is indicated in blue: P-h-D/E-Xaa-h-K/R-Xaa-K/R-(20-30 aa)-D/E-G-(4Xaa)-a-K/R-G ( $h$, hydrophobic; $X a a$, any amino acid; $a$, aromatic). Amino acid positions where the introns interrupt the coding region are indicated in red.
HHH015 Cases 1 and 2 and their father were obtained (data not shown). We detected no difference in the G3PDH expression pattern among patients, their parent and control (data not shown). Sequencing of all RT-PCR products demonstrated a homozygous state for all five patients and a heterozygous state for the parents (data not shown). Under similar RT-PCR conditions, OTC was amplified from human liver but not from the fibroblasts or lymphocytes (data not shown).

ORNT1 functional studies. We next studied whether the T32R change altered ORNT1 protein function using an established ornithine transport assay in transformed skin fibroblasts from control and $\mathrm{HHH}$ patients (6). Overexpression study results shown in Figure $3 A$ demonstrate that the T32R change does not completely eliminate ORNT1 function; instead, it retains a level of activity that is clearly less than the wild-type ORNT1 allele, but significantly more than the cells expressing the nonfunctional F188 allele (4). Moreover, inasmuch as the T32R mutation abolishes a theoretical PKC site and phosphorylation can occur on both serine and threonine residues within this consensus sequence, we were curious to see if a conservative T32S substitution would rescue the T32R ORNT1 mutation. Our results clearly show that the conservative T32S change restores ORNT1 function.

Concomitant with ornithine transport studies, we performed immunofluorescence using standard protocols to evaluate if the T32R mutation had any effect on the mitochondrial targeting pattern or protein stability of ORNT1 (6). Results shown in Figure 4 demonstrate that the ORNT1-T32R transporter has a normal mitochondrial targeting pattern as evidenced by its localization in multiple, elongated, cytoplasmic structures that coincide with the location of MitoTrackerlabeled mitochondria. Careful examination did not demonstrate staining of other cellular organelles. Experiments done with N-myc-ORNT1-T32S were indistinguishable from those using the wild-type and T32R ORNT1 transporters. In summary, regardless of ornithine transporting capability, both mutant constructs targeted normally to the mitochondria.

Lastly, using the ornithine transport assay, we examined if the partial retention of function of the ORNT1-T32R was reflected in the basal ornithine transport activity of primary untransfected fibroblasts obtained from HHH015 family members. For these experiments we used untransformed HHH015 fibroblasts and established transformed fibroblasts from $\mathrm{HHH}$ patients carrying the nonfunctional F188 $\Delta$ and E180K alleles. Surprisingly, results demonstrated that the HHH015 patients and untransfected HHH-F188D and HHH-E180K fibroblasts had similar basal ornithine transporting capability when compared with their respective controls (Fig. 3B).

Human ORNT2 analysis. Recognizing the phenotypic variability of our group of $\mathrm{HHH}$ patients, we sequenced the complete ORF of the ORNT2 gene, a potential modifier gene. Results of our analyses demonstrated that the patients with the mildest phenotype, HHH015 Cases 1 and 2, and their mother are carriers for a previously characterized ORNT2 gain of function polymorphism (Val181Gly) (6). This amino acid change occurs secondary to a $\mathrm{T}$ to $\mathrm{G}$ change at nt 542 of the ORNT2 ORF and eliminates a RsaI restriction site. Moreover, the HHH013 brothers and their parents carry the wild-type 
A

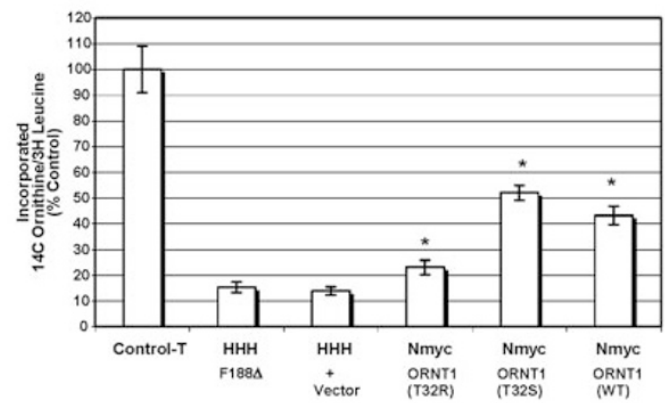

B

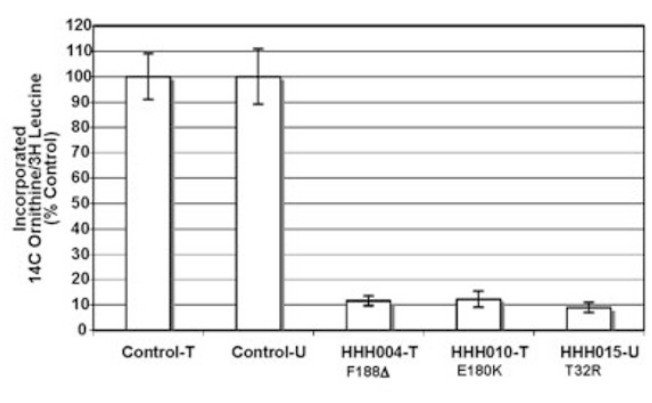

Figure 3. Ornithine transporting capacity of the Nmyc-ORNT1-T32R construct and cultured primary fibroblasts (HHH015) carrying the human ORNT1-T32R allele. Untransformed $(U)$ and transformed $(T)$ fibroblasts carrying different ORNT1 alleles (F188D, E180K, and T32R) were used to study their ability to incorporate ${ }^{14} \mathrm{C}$-ornithine and ${ }^{3} \mathrm{H}$-leucine $48 \mathrm{~h}$ after plating. The data are expressed as the ratio of $14 \mathrm{C} / 3 \mathrm{H}$ in precipitated protein and represent the mean \pm SEM. (A) We electroporated transformed HHH fibroblasts carrying the F188 $\Delta$ allele with $25 \mu \mathrm{g}$ of N-myc tagged construct [ORNT1-wild type (WT), ORNT1-T32R or ORNT1-T32S] or empty vector (pcDNA3.1). Graph shows comparison of the ornithine incorporation of untransfected control and untransfected HHH-F188 fibroblasts $v s$ transfected HHH-F188 $\Delta$ fibroblasts with either vector (pcDNA3.1), N-myc-ORNT1 (WT), N-myc-ORNT1-T32R, or N-myc-ORNT1-T32S. Data are pooled from three separate experiments with four measurements per data point per experiment. ${ }^{*} p<0.05 v s \mathrm{HHH}+$ vector. (B) Untransfected control and HHH fibroblasts were assayed for their ability to incorporate ornithine. Cells carrying the ORNT1 T32R (HHH015 Case 2), E180K, and F188 alleles were compared with their respective controls. Data are pooled from two separate experiments, representative of four additional experiments.

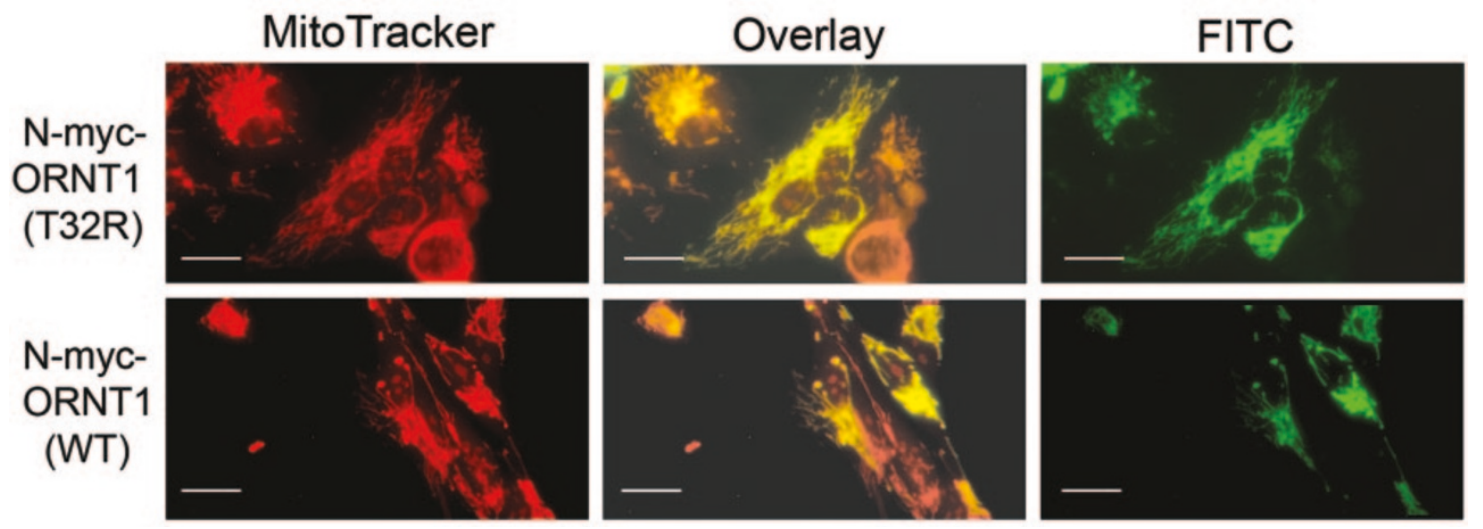

Figure 4. The human ORNT1-T32R transporter targets normally to the mitochondria. Transfected F188 cells were prelabeled with MitoTracker and processed for indirect immunofluorescence using a monoclonal FITC-labeled anti-myc antibody. The red images (left) show the pattern of MitoTracker labeling, characteristic of mitochondria, and the green images (right) show the mitochondrial localization of N-myc-ORNT1-T32R or N-myc-ORNT1-WT transporter. Center images show overlay of both red and green images. Transfection efficiency for these experiments was 25-30\%. Bars denote $25 \mu \mathrm{m}$.

ORNT2 allele. Subsequent RT-PCR analysis and follow-up RsaI restriction digests confirmed that both HHH015 siblings are carriers of the Gly ${ }^{181}$ allele (data not shown). Given our findings, we sought to determine whether the Gly ${ }^{181}$ polymorphism is prevalent in control groups that originally populated Mexico (11). Analysis of the ORNT2 gene in our Mayan Indian controls revealed a frequency of $36 \%$ for the Gly ${ }^{181}$ allele whereas, in the Spanish controls, only the wild-type $\mathrm{Val}^{181}$ allele was observed.

mtDNA haplogroup. Several studies suggest that different mitochondrial lineages or haplogroups may differ in their rates of ATP production and generation of reactive oxygen species (ROS), factors that could potentially affect mitochondrial function $(10,11)$. Given that ORNT1 is a mitochondrial protein and its deficiency could impact mitochondrial function, we sought to determine our patients' haplogroup and its potential influence on $\mathrm{HHH}$ clinical phenotype. Sequence analysis of the mtDNA control region (Fig. 5A) showed a large number of polymorphisms in both hypervariable regions of HHH015 Case 2; conversely, the HHH013 Case 3 sequence demonstrated only two polymorphisms. Subsequent comparative sequence analyses revealed that both HHH015 siblings belong to haplogroup A (North American Indian ancestry), whereas the HHH013 brothers are haplogroup $\mathrm{H}$, evident in $40 \%$ of individuals of European ancestry. AluI digestion of amplified cytochrome oxidase subunit I and tRNA-Ser genes further confirmed that the $\mathrm{HHH} 013$ patients are haplogroup $\mathrm{H}$ (Fig. 5B). Interestingly, the established diseased HHHF188 $\Delta$ fibroblasts used in our experiments, donated by a French Canadian patient, are haplogroup B (North American Indian ancestry) rather than of European descent.

\section{DISCUSSION}

The HHH syndrome is a disorder of the urea cycle and ornithine degradation pathway caused by mutations in the mitochondrial ornithine transporter, ORNT1 $(1,4)$. In this report, we describe five Mexican patients from two different, but related families who have a variable phenotype (Table 1). All five patients are homozygous for an ORNT1-T32R muta- 


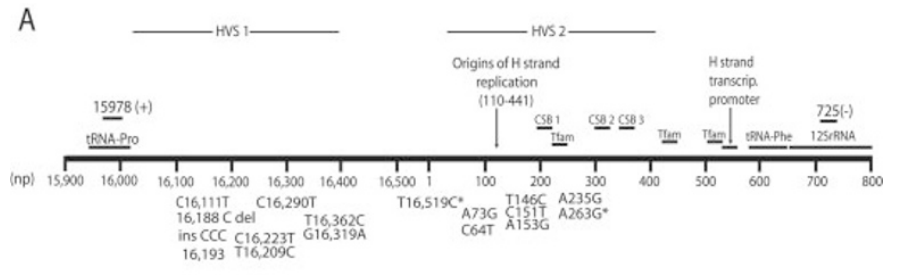

B
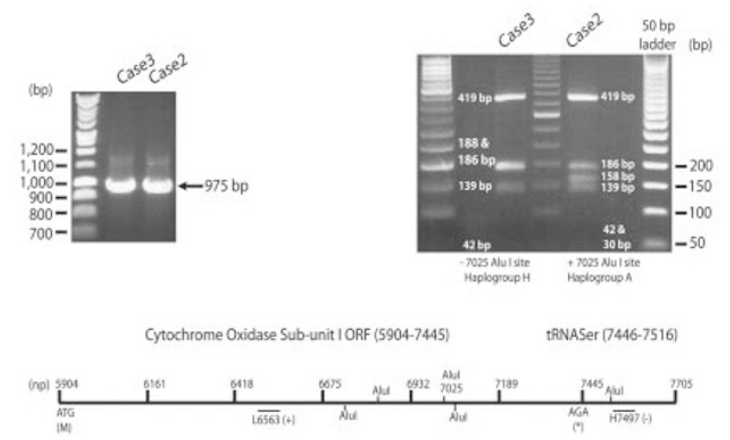

Figure 5. Summary of mtDNA control region polymorphisms in Case 2 (HHH015) and Case 3 (HHH013) and RFLP analysis of the 7025 AluI site. (A) Schematic representation of the mitochondrial control region (np 16024576) located between the tRNA-Pro and tRNA-Phe genes. Primers $15978(+)$ and $725(-)$ were used for amplification. Several key regions [hypervariable segment $(H V S)$, conserved sequence block $(C S B)$, and nuclear-encoded mitochondrial transcription binding sites (Tfam)] are shown in relation to the polymorphisms of both $\mathrm{HHH}$ patients. All nucleotide changes shown are observed in Case 2 (haplogroup A), whereas only the T16519 and A263G changes $(*)$ are observed in Case 3 (haplogroup H). (B) Amplification (left) and AluI digestion (right) of the cytochrome oxidase subunit 1/tRNA-Ser gene fragment to verify haplogroup $\mathrm{H}$ designation. The nucleotide change leading to the absence of the $7025 \mathrm{Alu} \mathrm{I}$ site occurs in the third base of the Ala (375) codon and is present in the European haplogroup H. Schematic drawing of amplified region showing multiple AluI sites (bottom).

tion that occurs in the first hydrophilic loop facing the mitochondrial matrix at a conserved threonine (Fig. 2). This conserved threonine is included in the mitochondrial signature of all MCF proteins and predicts a theoretical PKC phosphorylation site (Ser/Thr-Xaa-Arg/Lys). Interestingly, recent studies demonstrate that the phosphate and ADP/ATP mitochondrial transporters are phosphorylated thus suggesting that phosphorylation may play a role in the physiology of oxidative phosphorylation or perhaps other metabolic pathways (14).

Cultured HHH fibroblasts not only define the cellular phenotype in $\mathrm{HHH}$ syndrome but can also be used to study the functional consequences of ORNT1 mutations and mechanisms of disease $(4,6)$. Transient overexpression studies of the ORNT1-T32R allele revealed normal targeting to the mitochondria and a significant level of function relative to the F188 $\Delta$ cells transfected with an empty vector (Fig. $3 A$ and Fig. 4). This latter finding proved interesting since the basal ornithine transport of primary untransfected HHH015 fibroblasts exhibited an activity level similar to untransfected fibroblasts carrying nonfunctional alleles such as the R179ter (unpublished observations), F188 $\Delta$ and E180K (Fig. 3B). These observations are important since they confirm a previously reported discordance between the clinical and cellular phenotype in patients with HHH syndrome (15). Lastly, transient transfection experiments also demonstrated that a con- servative T32S mutation exhibited ornithine incorporation activity comparable to the wild-type ORNT1 allele vis-à-vis the deficient transport rendered by the ORNT1-T32R allele (Fig. 3A). Inasmuch as both serine and threonine may serve as phosphorylation residues within the theoretical mutated PKC consensus sequence, these results suggest that phosphorylation may perhaps be an important regulator of ORNT1 function (13). Future studies will explore this intriguing observation.

Our study of two related families clearly demonstrates that a single ORNT1 genotype, T32R, can have different clinical presentations and course (Table 1). In our study, the factors underlying the differences in cognitive development and school performance among patients of both affected families is unknown, although we cannot definitely rule out a history of more subtle and unrecognized hyperammonemic episodes. Of special interest is the HHH013 family that has three affected siblings and three unaffected siblings sharing a similar genetic background and environment. Surprisingly, all the brothers have a history of academic difficulties but IQ evaluation was only performed on the siblings with the HHH syndrome. Although the mild mental retardation in the oldest affected sibling (Case 3) was most likely due to the HHH syndrome, we are unable to make judgments on the relative contribution of the HHH syndrome to the low IQ in the remaining $\mathrm{HHH}$ brothers in this family. The reason for the late deterioration and death of the oldest affected sibling (Case 3) in the HHH013 family in contrast to the stable clinical course of his younger affected siblings is perplexing. Recent reports describe a group of French Canadian patients with the ORNT1F188 $\Delta$ allele that exhibit a variable phenotype ranging from neonatal onset hyperammonemia to a mild adult presentation (Shih VE et al, Genotype and phenotype findings in the hyperornithinemia-hyperammonemia-homocitrullinuria (HHH) syndrome, VIIIth International Conference on Inborn Errors of Metabolism, 13-17 September 2000, Cambridge, UK). Interestingly, a different cohort of French Canadian HHH patients from three different families that have a common ancestor presented with different phenotypes that included neonatal death, coagulation abnormalities, and low normal intelligence to severe mental retardation (1).

One potential modifier gene in $\mathrm{HHH}$ syndrome is the second mitochondrial ornithine transporter, ORNT2. Human ORNT2 is a functional retroposon expressed in liver and fibroblasts that can rescue the deficient ornithine transport in cultured fibroblasts when overexpressed (6). Protein polymorphisms in ORNT2 that could further enhance or decrease ornithine transport may potentially influence the phenotype in our group of patients. Our work demonstrates that the two least affected patients, HHH015 Cases 1 and 2, are carriers for the ORNT2-Gly ${ }^{181}$ polymorphism, a gain of function mutation; whereas the HHH013 brothers carry the wild-type ORNT2-Val ${ }^{181}$ allele. At first glance, these results suggest a correlation between the ORNT2 genotype and the HHH phenotype of our patients. However, we are limited in our ability to make conclusions since it is not known if the ORNT2 gene is expressed in the periportal hepatocytes where the urea cycle enzymes are found $(1,6)$. Future studies of additional $\mathrm{HHH}$ 
patients carrying identical ORNT1 mutations will further elucidate the influence of human ORNT2 on HHH syndrome phenotype. Taken together, our observations raise the question as to what modifying factors may be hampering the ornithine transport in primary HHH015 cultured fibroblasts that exhibit significant residual function in overexpression studies as well as express a redundant gene with a gain of function polymorphism. To begin to address this question, we explored the background mitochondrial physiology of our patients.

Whether different mitochondrial lineages can contribute to the cellular pathophysiology in HHH syndrome or other urea cycle disorders is not known. Recent studies using cultured astrocytes suggest that elevated ammonia and glutamine, a hallmark of HHH syndrome and other urea cycle disorders, may activate the mitochondrial permeability transition pore secondary to ROS generation (16). Haplogroups, which represent shared mtDNA mutations in the control region, tend to show regional specificity and are theoretically associated with functional changes in oxidative phosphorylation $(10,11)$. Results of our study demonstrate that the HHH015 siblings are of American Indian descent (i.e. Mayan Indian) whereas the HHH013 brothers are of European ancestry (Fig. 5). Although these results do not allow us to draw conclusions regarding the differences in phenotype between families, it does provide a framework for the selection of appropriate controls in future experiments.

Taken together, our work and that of others strongly suggest that the genotype of HHH patients cannot predict the clinical course of the disease. It appears that other potential modifying factors such as gene redundancy, mitochondrial background and/or perhaps other as yet unidentified factors could further influence the phenotype of HHH patients (17).

Acknowledgments. The authors thank Drs. Grant Mitchell (Ste. Justine Hospital) and Dave Valle (Johns Hopkins University) for the generous gift of HHH fibroblast cell lines and French Canadian control DNAs. All other control DNAs were a generous gift from Dr. John Harley (OMRF) and Dr. Doug Wallace (UCI). We also thank Sheryl Christofferson and Greg Merrick for performing all the sequencing.

\section{REFERENCES}

1. Valle D, Simell O 2001 The hyperornithinemias. In: Scriver CR, Beaudet AL, Sly WS, Valle D (eds) The Metabolic and Molecular Basis of Inherited Disease. McGraw Hill, New York, pp 1857-1896

2. Brusilow SW, Horwich AL 2001 Urea cycle enzymes. In: Scriver CR, Beaudet AL, Sly WS, Valle D (eds) The Metabolic and Molecular Basis of Inherited Disease. McGraw Hill, New York, pp 1909-1964

3. Kaplan RS 1996 Mitochondrial transport processes. In: Schultz SG, Andreoli TE, Brown AM, Fambrough DM, Hoffman JF, Welsh MJ (eds) Molecular Biology of Membrane Transport Disorders. Plenum Press, New York, pp 277-302

4. Camacho JA, Obie C, Biery B, Goodman BK, Hu CA, Almashanu S, Steel G, Casey R, Lambert M, Mitchell GA, Valle D 1999 Hyperornithinaemia-hyperammonaemiahomocitrullinuria syndrome is caused by mutations in a gene encoding a mitochondrial ornithine transporter. Nat Genet 22:151-158

5. Fiermonte G, Dolce V, David L, Santorelli FM, Dionisi-Vici C, Palmieri F, Walker JE 2003 The mitochondrial ornithine transporter. Bacterial expression, reconstitution, functional characterization, and tissue distribution of two human isoforms. J Biol Chem 278:32778-32783

6. Camacho JA, Rioseco-Camacho N, Andrade D, Porter J, Kong J 2003 Cloning and characterization of human ORNT2: a second mitochondrial ornithine transporter that can rescue a defective ORNT1 in patients with the hyperornithinemiahyperammonemia-homocitrullinuria syndrome, a urea cycle disorder. Mol Genet Metab 79:257-271

7. Tsujino S, Kanazawa N, Ohashi T, Eto Y, Saito T, Kira J, Yamada T 2000 Three novel mutations (G27E, insAAC, R179X) in the ORNT1 gene of Japanese patients with hyperornithinemia, hyperammonemia, and homocitrullinuria syndrome. Ann Neurol 47:625-631

8. Korman SH, Kanazawa N, Abu-Libdeh B, Gutman A, Tsujino S 2004 Hyperornithinemia, hyperammonemia, and homocitrullinuria syndrome with evidence of mitochondrial dysfunction due to a novel SLC25A15 (ORNT1) gene mutation in a Palestinian family. J Neurol Sci 218:53-58

9. Salvi S, Santorelli FM, Bertini E, Boldrini R, Meli C, Donati A, Burlina AB, Rizzo C, Di Capua M, Fariello G, Dionisi-Vici C 2001 Clinical and molecular findings in hyperornithinemia-hyperammonemia-homocitrullinuria syndrome. Neurology 57:911-914

10. Ruiz-Pesini E, Mishmar D, Brandon M, Procaccio V, Wallace DC 2004 Effects of purifying and adaptive selection on regional variation in human mtDNA. Science 303:223-226

11. Wallace DC 2005 A mitochondrial paradigm of metabolic and degenerative diseases, aging, and cancer: a dawn for evolutionary medicine. Annu Rev Genet 39:359-407

12. Andrews RM, Kubacka I, Chinnery PF, Lightowlers RN, Turnbull DM, Howell N 1999 Reanalysis and revision of the Cambridge reference sequence for human mitochondrial DNA. Nat Genet 23:147

13. Toomik R, Ek P 1997 A potent and highly selective peptide substrate for protein kinase C assay. Biochem J 322:455-460

14. Schulenberg B, Aggeler R, Beechem JM, Capaldi RA, Patton WF 2003 Analysis of steady-state protein phosphorylation in mitochondria using a novel fluorescent phosphosensor dye. J Biol Chem 278:27251-27255

15. Tuchman M, Knopman DS, Shih VE 1990 Episodic hyperammonemia in adult siblings with hyperornithinemia, hyperammonemia, and homocitrullinuria syndrome. Arch Neurol 47:1134-1137

16. Rama Rao KV, Jayakumar AR, Norenberg MD 2005 Role of oxidative stress in the ammonia-induced mitochondrial permeability transition in cultured astrocytes. Neurochem Int 47:31-38

17. Dipple KM, McCabe ER 2000 Phenotypes of patients with "simple" Mendelian disorders are complex traits: thresholds, modifiers, and systems dynamics. Am J Hum Genet 66:1729-1735 